\title{
The influence of omniscient technology on cryptography
}

\section{Weihong Huang, Jian Li}

Weihong Huang, Jian $\mathrm{Li}$, "The influence of omniscient technology on cryptography," Proc. SPIE 7490, PIAGENG 2009: Intelligent Information, Control, and Communication Technology for Agricultural Engineering, 749023 (10 July 2009); doi: 10.1117/12.836819

Event: International Conference on Photonics and Image in Agriculture Engineering (PIAGENG 2009), 2009, Zhangjiajie, China 


\section{The influence of omniscient technology on cryptography (Withdrawal Notice)}

Proc. SPIE 7490, 749023 (2009); http://dx.doi.org/10.1117/12.836819

Online Publication Date: 10 July 2009

Withdrawn from Publication: 13 November 2015

Conference Date: 11-12 July 2009

Conference Location: Zhangjiajie, China

Conference Title: PIAGENG 2009: Intelligent Information, Control, and Communication Technology for Agricultural Engineering

Conference Chairs: Honghua Tan, Qi Luo

Weihong Huang

Hunan Univ. of Science and Technology (China)

J ian Li

Hunan Univ. (China)

This paper has been identified by SPIE as fraudulent and was withdrawn on 13 November 2015.

PIAGENG 2009: Intelligent Information, Control, and Communication Technology 